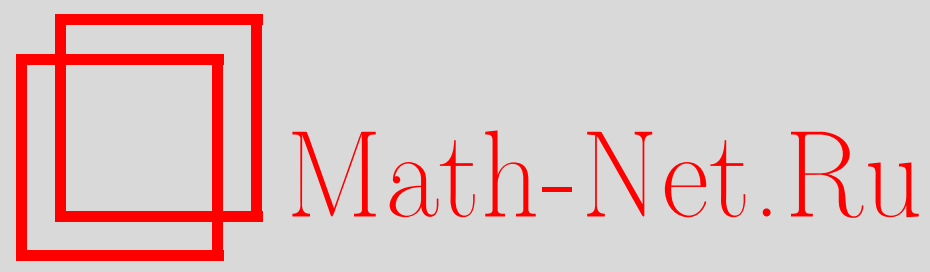

Л. Е. Базилевич, Топология гиперпространства выпуклых тел постоянной ширины, Матем. заметки, 1997, том 62, выпуск 6, 813-819

DOI: https://doi.org/10.4213/mzm1670

Использование Общероссийского математического портала Math-Net.Ru подразумевает, что вы прочитали и согласны с пользовательским соглашением http://www . mathnet.ru/rus/agreement

Параметры загрузки:

IP : 34.229 .108 .108

26 апреля 2023 г., 10:04:44 


\title{
ТОПОЛОГИЯ ГИПЕРПРОСТРАНСТВА ВЫПУКЛЫХ ТЕЛ ПОСТОЯННОЙ ШИРИНЫ
}

\section{Л. Е. Базилевич}

\begin{abstract}
Доказано, что гиперпространство всех выпуклых тел постоянной ширины в евклидовом пространстве $\mathbb{R}^{n}, n \geqslant 2$, гомеоморфно стягиваемому $Q$-многообразию (через $Q$ обозначается гильбертов куб). В доказательстве используется явно построенная ретракция всего гиперпространства выпуклых тел на гиперпространство выпуклых тел постоянной шшрины.
\end{abstract}

Библиограбоя: 4 названия.

Исследование гиперпространства выпуклых компактов методами бесконечномерной топологии началось с работы [1], в которой доказано, что гиперпространство вьпуклых компактов в евклидовом пространстве $\mathbb{R}^{n}$ при $n \geqslant 2$ гомеоморфно дополнению точки в гильбертовом кубе

$$
Q=\prod_{i=1}^{\infty} I_{i} .
$$

Этот результат открыл возможность для описания топологии гиперпространств компактных выпуклых тел, обладающих некоторыми дополнительньми геометрическими свойствами [2], [3]. В настоящей работе рассмотрено гиперпространство вьпуклых тел постоянной ширины в $\mathbb{R}^{n}$. Полученньй результат является аналогом упоминаемого выше результата из [1]. Прежде чем переходить к его формулировке, приведем необходимые определения.

Проективное пространство $\mathbb{R P}^{n-1}$ рассматриваем как пространство прямых, проходящих через начало координат в $\mathbb{R}^{n}$. Говорим, что прямая, отрезок, луч и т.п. в $\mathbb{R}^{n}$ имеют направление $l \in \mathbb{R P}^{n-1}$, если они параллельны $l$.

Рассмотрим произвольное направление $l$. Каждое компактное вьпуклое тело расположено между двумя опорными гиперплоскостями, ортогональными $l$. Расстояние между этими гиперплоскостями обозначим через $\operatorname{diam}(A, l)$. Тело $A$ называется телом постоянной иирины $d$, если $\operatorname{diam}(A, l)=\operatorname{diam} A=d$ для всех $l$.

Обозначим через $\mathscr{M}$ гиперпространство всех выпуклых тел в евклидовом пространстве $\mathbb{R}^{n}$; оно наделяется метрикой Хаусдорфа

$$
d(A, B)=\inf \left\{\varepsilon>0 \mid A \subset O_{\varepsilon}(B), B \subset O_{\varepsilon}(A)\right\}, \quad A, B \in \mathscr{M} .
$$

Через $\mathscr{N}$ обозначим подпространство в $\mathscr{M}$, состоящее из всех выпуклых тел постоянной ширины. 
ТЕОРемА. При $n \geqslant 2$ гиперпространство $\mathcal{N}$ гомеоморфно стягиваемому $Q$-многообразию.

Доказательство этой теоремы основано на следуюшей характеризационной теореме Торуньчика для $Q$-многообразий.

Tеорема [4]. Локально компактное пространство $X \in \mathrm{ANR}$ гомеоморфно $Q$-многообразию, если и только если оно удовлетворяет свойству дизбюнктной аппроксимачии.

Здесь свойство дизғюнктной аппроксимаиии означает, что для каждой непрерывной функции $\varepsilon: X \rightarrow(0,+\infty)$ существуют непрерьвные отображения $f_{\varepsilon}, g_{\varepsilon}: X \rightarrow X$ такие, что $f_{\varepsilon}(X) \cap g_{\varepsilon}(X)=\varnothing$ и $d\left(f_{\varepsilon}(x), x\right)<\varepsilon(x), d\left(g_{\varepsilon}(x), x\right)<\varepsilon(x)$.

Нам понадобятся некоторые свойства вьпуклых тел постоянной ширины. Доказательства наиболее простых из них предоставляются читателю. Пусть $A$ - выпуклое тело постоянной ширины $d>0$. Через $w_{i}(A, l), i=1,2$, обозначим опорные гиперплоскости тела $A$ в направлении $l$.

ПРЕДЛОЖЕНИЕ 1. Множества $w_{i}(A, l) \cap A, i=1,2$, являются точками для произвольного направления $l$. Отрезок, соединяющий эти точки, имеет направление $l$.

В дальнейшем, отрезок, о котором идет речь в предложении 1 , называется $\partial и а м е т-$ ром тела $A$ в направлении l.

ПРЕДЛОЖЕНИЕ 2. Для произвольной точки $a \in \mathrm{Bd} A$ существует направление $l$ такое, что одна из опорных әиперплоскостей $\kappa$ телу $A$ в направлении $l$ пересекается $c$ A по точке $а$.

Через $C(A)$ обозначим множество точек $a \in A$, принадлежаших более чем одному диаметру тела $A$.

ПРЕДЛОЖЕНИЕ 3. Ecли $d(C(A), \operatorname{Bd} A)>0$, mo отображсение $p: \operatorname{Bd} A \rightarrow \exp A$, ставящее в соответствие каждой точке $а \in \operatorname{Bd} A$ диаметр тела $A$ с кониом в этой точке, является непрерывным.

ПРЕДЛОЖЕНИЕ 4. Тело постоянной ииринь является обгединением всех своих диаметров.

ДокАЗАтЕльство. Пусть $\left[b_{l}, c_{l}\right]$ - диаметр направления $l$ тела $A$ и $T=\cup\left\{\left[b_{l}, c_{l}\right]\right.$ | $\left.l \in \mathbb{R} \mathbb{P}^{n-1}\right\}$. Предположим, что существует точка $a \in A \backslash T$. Поскольку $T$ - замкнутое множество и $\mathrm{Bd} A \subset T$, существует $\varepsilon>0$ такое, что $K(a, \varepsilon) \subset A \backslash T$. Пусть $d \in \operatorname{Bd} A-$ точка, в которой реализуется $\max \left\{d\left(a, d^{\prime}\right) \mid d^{\prime} \in \operatorname{Bd} A\right\}$, a $l_{0}$-направлениепрямой $(a, d)$. Очевидно, что $d(a, d) \geqslant \frac{1}{2} \operatorname{diam} A$. Пусть $\left[b_{l_{0}}, c_{l_{0}}\right]$ - диаметр направления $l_{0}$, причем его конщы обозначены так, что $\left[b_{l_{0}}, d\right] \cap\left[c_{l_{0}}, a\right]=\varnothing$. Из определения точки $d$ вытекает, что $d\left(b_{l_{0}}, a\right) \leqslant d(d, a)$. Но тогда легко видеть, что $d\left(c_{l_{0}}, d\right)>d\left(c_{l_{0}}, b_{l_{0}}\right)=\operatorname{diam} A$. Это противоречие показывает, что $A=T$.

Укажем универсальный способ построения вьпуклых тел постоянной ширины в $\mathbb{R}^{n}$. Зафиксируем счетную всюду плотную последовательность $\left(l_{i}\right)_{i=1}^{\infty}$ направлений $l_{i} \in \mathbb{R P}^{n-1}$. Методом математической индукции по $k$ построим выпуклое тело $A$ постоянной ширины $d>0$. 
Пусть $k=1$. Зафиксируем произвольньй отрезок $\left[a_{1}, b_{1}\right]$ длины $d$ и направления $l_{1}$. Положим $T\left(a_{1}, b_{1}\right)=\mathrm{Cl}\left(K\left(a_{1}, d\right) \cap K\left(b_{1}, d\right)\right)$. Легко видеть, что $T\left(a_{1}, b_{1}\right)$ - вьпуклое тело ширины не меньше $d$, т.е. $\operatorname{diam}\left(T\left(a_{1}, b_{1}\right), l\right) \geqslant d$ для всех $l \in \mathbb{R}^{n}$ и $\operatorname{diam}\left(T\left(a_{1}, b_{1}\right), l_{1}\right)=d$. Положим $A_{1}=T\left(a_{1}, b_{1}\right)$.

Пусть $k=2$. Отрезок $\left[a_{2}, b_{2}\right]$ длины $d$ и направления $l_{2}$ выберем так, чтобы он удовлетворял условию $\left[a_{2}, b_{2}\right] \subset T\left(a_{1}, b_{1}\right)$. Легко видеть, что при этом $\left[a_{1}, b_{1}\right] \subset T\left(a_{2}, b_{2}\right)$. Множество $A_{2}=A_{1} \cap T\left(a_{2}, b_{2}\right)$ является вьпуклым телом ширины не меньше $d$ и $\operatorname{diam}\left(A_{2}, l_{j}\right)=d$ при $j=1,2$.

Предположим, что построение уже осуществлено для $k=1,2, \ldots, n-1$, и проведем его для $k=n$. Отрезок $\left[a_{n}, b_{n}\right]$ длины $d$ и направления $l_{n}$ выберем из условия $\left[a_{n}, b_{n}\right] \subset A_{n-1}$. Это возможно, поскольку $A_{n-1}$ - вьпуклое тело ширины не меньше $d$. Положим $A_{n}=A_{n-1} \cap T\left(a_{n}, b_{n}\right)$. Тогда $A_{n}$ - вьпуклое тело ширины не меньше $d$ и $\operatorname{diam}\left(A_{n}, l_{j}\right)=d$ для $j=1,2, \ldots, n$.

Положим

$$
A=\bigcap_{i=1}^{\infty} A_{i}=\bigcap_{i=1}^{\infty} T\left(a_{i}, b_{i}\right) .
$$

Множество $A$ является выпуклым телом постоянной ширины $d$, причем

$$
A=\mathrm{Cl}\left(\bigcup_{i=1}^{\infty}\left[a_{i}, b_{i}\right]\right)
$$

Легко видеть, что таким образом мы можем получить все выпуклые тела постоянной ширины. Сделаем несколько замечаний к построению.

ПРЕДЛОЖЕНИЕ 5. Имеет место равномерная непрерывность построения, т.е. для каждого $\varepsilon>0$ существует $n_{\varepsilon} \in \mathbb{N}$ такое, ито для произвольных двух выпуклых тел

$$
A^{\prime}=\mathrm{Cl}\left(\bigcup_{i=1}^{\infty}\left[a_{i}^{\prime}, b_{i}^{\prime}\right]\right), \quad A^{\prime \prime}=\mathrm{Cl}\left(\bigcup_{i=1}^{\infty}\left[a_{i}^{\prime \prime}, b_{i}^{\prime \prime}\right]\right)
$$

постоянной ширины $d$ с условием $\left[a_{i}^{\prime}, b_{i}^{\prime}\right]=\left[a_{i}^{\prime \prime}, b_{i}^{\prime \prime}\right]$ для $i=1, \ldots, n_{\varepsilon}$ выполнено $d\left(A^{\prime}, A^{\prime \prime}\right)<\varepsilon d$.

ПРЕДЛОЖЕНИЕ 6. Пусть

$$
A=\bigcap_{i=1}^{\infty} T\left(a_{i}, b_{i}\right)
$$

- выпуклое тело постоянной ширины d такое, что $d(C(A), \mathrm{Bd} A) \geqslant \varepsilon>0$. Тогда для произвольного номера $n$ существует угол $\theta(\varepsilon, n), \theta(\varepsilon, n) \rightarrow 0$ nри $n \rightarrow \infty$ такой, что произвольные отрезки вида $\left[a_{j}, c\right],\left[b_{j}, c\right]$ длины $d$ и направления, отличающегося от направления отрезка $\left[a_{j}, b_{j}\right]$ на угол не больший $\theta(\varepsilon, n)$, принадлежат множеству

$$
A_{n}=\bigcap_{i=1}^{n} T\left(a_{i}, b_{i}\right) .
$$


ПРЕДЛОЖЕНИЕ 7. Пусть $B$ - выпуклое тело ширины не меньие $d$. Для произвольного отрезка $[a, b]$ длины $d^{\prime} \leqslant d$ существует единственный отрезок $\left[a^{\prime}, b^{\prime}\right] \subset B$ той же длины и направления, что $u[a, b]$, являющийся ближайшим $\kappa[a, b]$ в метрике Хаусдорфа. Соотношение $[a, b] \mapsto\left[a^{\prime}, b^{\prime}\right]$ непрерывно зависит от $B$.

В дальнейшем условимся считать, что концы диаметров одного направления обозначены с учетом ориентации направления, а именно: предполагаем, что диаметры $\left[a^{\prime}, b^{\prime}\right]$ и $\left[a^{\prime \prime}, b^{\prime \prime}\right]$ выпуклых тел $A^{\prime}$ и $A^{\prime \prime}$ соответственно обозначены так, что $\left[a^{\prime}, a^{\prime \prime}\right] \cap\left[a^{\prime \prime}, b^{\prime \prime}\right]=\varnothing$.

Возвращаемся к доказательству теоремы. Пусть $\varepsilon: \mathscr{N} \rightarrow(0,+\infty)$ - непрерьвная функция. Для произвольного $A \in \mathscr{N}$ положим $f_{\varepsilon}(A)=\left\{x \in \mathbb{R}^{n} \mid d(x, A) \leqslant \varepsilon(A)\right\}$. Легко видеть, что $f_{\varepsilon}(A)$ - вьпуклое тело постоянной ширины $\operatorname{diam} A+2 \varepsilon(A)$. Имеем $d\left(A, f_{\varepsilon}(A)\right)=\varepsilon$ и, кроме того, $d\left(\operatorname{Bd}\left(f_{\varepsilon}(A)\right), C\left(f_{\varepsilon}(A)\right)\right) \geqslant \varepsilon(A)$ для произвольного $A \in \mathcal{N}$.

Построим отображение $g_{\varepsilon}$. Пусть $\varepsilon^{\prime}=\varepsilon / 2$. Произвольное $f_{\varepsilon^{\prime}}(A)$ можно представить в виде

$$
\mathrm{Cl}\left(\bigcup_{i=1}^{\infty}\left[a_{i}, b_{i}\right]\right)
$$

как описано вьше. Ясно, что $d\left(\mathrm{id}, f_{\varepsilon^{\prime}}\right)=\varepsilon^{\prime}$.

Для выпуклого тела $f_{\varepsilon^{\prime}}(A)$ и для числа $\delta(A)=\varepsilon(A) /(2 \operatorname{diam} A)$ выберем номер $n_{\delta}(A)$ из предложения 5. Из предложения 6 для номера $n_{\delta(A)}$ получим число $\theta\left(\varepsilon(A) / 2, n_{\delta(A)}\right)$. Выберем произвольную непрерьвную функцию $t: \mathcal{N} \rightarrow(0, \pi / 2)$ такую, что $0<t(A)<$ $\theta\left(\varepsilon(A) / 2, n_{\delta(A)}\right), A \in \mathcal{N}$. Положим $G_{1}(A)=\bigcap\left\{T\left(a_{1}, c\right) \mid d\left(a_{1}, c\right)=d\left(a_{1}, b_{1}\right)\right.$ и угол $\angle c a_{1} b_{1}$ не больше $\left.t(A)\right\}$. Из сказанного выше легко видно, что $\left[a_{j}, b_{j}\right] \subset G_{1}(A)$ для $j=2, \ldots, n_{\delta(A)}$, поэтому полагаем

$$
G_{2}(A)=G_{1}(A) \cap T\left(a_{2}, b_{2}\right), \ldots, \quad G_{n_{\delta(A)}}(A)=G_{n_{\delta(A)}-1}(A) \cap T\left(a_{n_{\delta(A)}}, b_{n_{\delta(A)}}\right) .
$$

Очевидно, что все $G_{j}(A), j=1, \ldots, n_{\delta(A)},-$ вьпуклые тела ширины не меньше $\operatorname{diam} A+$ $\varepsilon(A)$.

Для следующих шагов $n_{\delta(A)}+k, k=1,2, \ldots$, выберем диаметры $\left[a_{n_{\delta(A)}^{\prime}+k}^{\prime}, b_{n_{\delta(A)}+k}^{\prime}\right]$ так, чтобы они были ближайшими к диаметрам $\left[a_{n_{\delta(A)}}+k, b_{n_{\delta(A)}}+k\right]$ и принадлежали выпуклому телу

$$
G_{n_{\delta(A)}+k-1}(A)=G_{n_{\delta(A)}}(A) \cap \bigcap_{i=n_{\delta(A)}+1}^{n_{\delta(A)}+k-1} T\left(a_{i}^{\prime}, b_{i}^{\prime}\right)
$$

(см. предложение 7). Положим

$$
g_{\varepsilon}(A)=\bigcap_{i=1}^{\infty} G_{i}(A)
$$

Легко видеть, что $g_{\varepsilon}(A)$ - вьпуклое тело постоянной ширины и $d\left(A, g_{\varepsilon}(A)\right) \leqslant \varepsilon(A) / 2+$ $\delta(A) \operatorname{diam} A=\varepsilon(A)$. Поскольку $a_{1} \in C\left(g_{\varepsilon}(A)\right)$, то $d\left(\operatorname{Bd}\left(g_{\varepsilon}(A)\right), C\left(g_{\varepsilon}(A)\right)\right)=0$. Отсюда вытекает, что $d\left(g_{\varepsilon}, \mathrm{id}\right)<\varepsilon$ и $f_{\varepsilon}(\mathscr{N}) \cap g_{\varepsilon}(\mathscr{N})=\varnothing$. Следовательно, пространство $\mathscr{N}$ удовлетворяет условию дизъюнктной апшроксимации. 
Покажем, что гиперпространство $\mathscr{N}$ является ретрактом гиперпространства $\mathscr{M}$. Для произвольного вьпуклого тела $A$ сушествует замкнутьй шар $K \subset A$ наибольшего радиуса $r(A)$, причем возможно неединственный. Но среди всех таких шаров единственньй является ближайшим к $A$ в метрике Хаусдорфа. Обозначим через $d(A)$ максимальный диаметр всех вьпуклых тел постоянной ширины $B$, содержащихся в $A$. Очевидно, что $d(A) \geqslant 2 r(A)$. Однако, среди всех выпуклых тел постоянной ширины $d(A)$ расстояние Хаусдорфа к $A$ реализуется не единственным образом.

Зафиксируем произвольное $A \in \mathscr{M}$. Через $\mathscr{L}(A)$ обозначим множество всех вьпуклых тел $B \subset A$ постоянной ширины $d(A)$. Для каждого $B \in \mathscr{L}(A)$ обозначим через $V\left(B, l_{1}\right)$ центр диаметра направления $l_{1}$. Положим $V\left(l_{1}\right)=\left\{V\left(B, l_{1}\right) \mid B \in \mathscr{L}(A)\right\}$.

ЛЕмМА. $V\left(l_{1}\right)$ является ограниченным замкнутым выпуклым множеством без внутренних точек, т.е. не более чем $(n-1)$-мерным.

ДокАЗАТЕЛЬСТво. Очевидно, что множество $V\left(l_{1}\right)$ ограничено и имеет пустую внутренность. Покажем замкнутость этого множества. Пусть $\left\{M_{i}\right\}_{i=1}^{\infty}, M_{i} \in V\left(l_{1}\right),-$ произвольная сходящаяся в $A$ последовательность. Обозначим через $\left\{\left[N_{i}^{1}, L_{i}^{1}\right]\right\}_{i=1}^{\infty}$ последовательность сегментов $\left[N_{i}^{1}, L_{i}^{1}\right]$ направления $l_{1}$ и длины $d(A)$, центрами которых являются точки $M_{i}$. Фиксируем произвольное $B_{i} \in \mathscr{L}(A)$ такое, что $\left[N_{i}^{1}, L_{i}^{1}\right]$ является для $B_{i}$ диаметром направления $l_{1}$. Из замкнутости $A$ вытекает, что сегмент

$$
\left[N^{1}, L^{1}\right]=\lim _{i \rightarrow \infty}\left[N_{i}^{1}, L_{i}^{1}\right]
$$

принадлежит телу $A$ и его центр - точка $M$ - является пределом последовательности $\left\{M_{i}\right\}_{i=1}^{\infty}$. Построение вьпуклого тела $B \in \mathscr{L}(A)$ с диаметром $\left[N^{1}, L^{1}\right]$ будем проводить по индукции.

Вовсех телах $B_{i}$ проведем диаметры $\left[N_{i}^{2}, L_{i}^{2}\right]$ направления $l_{2}$. Из последовательности $\left\{\left[N_{i}^{2}, L_{i}^{2}\right]\right\}_{i=1}^{\infty}$ выберем сходящуюся подпоследовательность $\left\{\left[N_{k_{i}^{2}}^{2}, L_{k_{i}^{2}}^{2}\right]\right\}_{i=1}^{\infty}$. Очевидно, что

$$
\lim _{i \rightarrow \infty}\left[N_{k_{i}^{2}}^{2}, L_{k_{i}^{2}}^{2}\right]=\left[N^{2}, L^{2}\right]
$$

принадлежит $A$. Предположим, что сегменты $\left[N^{j}, L^{j}\right]$ направлений $l_{j}, j \geqslant 2$, уже построены. Через $\left[N^{j+1}, L^{j+1}\right]$ обозначим предел сходящейся подпоследовательности $\left\{\left[N_{k_{i}^{j+1}}^{j+1}, L_{k_{i}^{j+1}}^{j+1}\right]\right\}_{i=1}^{\infty}$ последовательности $\left\{\left[N_{k_{i}^{j}}^{j+1}, L_{k_{i}^{j+1}}^{j+1}\right]\right\}_{i=1}^{\infty}$ диаметров направления $l^{j+1}$ выпуклых тел $B_{k_{i}^{j}}$. Легко видеть, что вьпуклое тело

$$
B=\lim _{i \rightarrow \infty} B_{k_{i}^{i}}=\operatorname{Cl}\left(\cup\left\{\left[N^{i}, L^{i}\right] \mid i=1,2, \ldots\right\}\right)
$$

является искомым.

Теперь покажем вьпуклость множества $V\left(l_{1}\right)$. Пусть $M_{1}, M_{2} \in V\left(l_{1}\right)$. Достаточно показать, что середина $M_{3}$ отрезка $\left[M_{1}, M_{2}\right]$ также принадлежит множеству $V\left(l_{1}\right)$, т.е. существует вьпуклое тело $B^{*} \in \mathscr{L}(A)$, имеющее диаметр $\left[N_{3}, L_{3}\right]$ в направлении $l_{1}$, серединой которого является точка $M_{3}$.

Пусть $\left[N_{k}^{1}, L_{k}^{1}\right], k=1,2,-$ соответствующие сегменты длины $d(A)$, середины которых находятся в точках $M_{k}$. Зафиксируем некоторые вьпуклые тела $B_{k} \subset \mathscr{L}(A), k=1,2$, для которых сегменты $\left[N_{k}^{1}, L_{k}^{1}\right]$ являются диаметрами направления $l_{1}$. 
Для произвольного $j=2,3, \ldots$ обозначим через $\left[N_{k}^{j}, L_{k}^{j}\right], k=1,2$, диаметры тел $B_{k}$ направления $l_{j}$. Будем предполагать, что буквы $N$ и $L$ употребляются согласованно, т.е. всегда $\left[N_{1}^{j}, N_{2}^{j}\right] \cap\left[L_{1}^{j}, L_{2}^{j}\right]=\varnothing$. Обозначим через $N_{3}^{j}$ и $L_{3}^{j}$ середины отрезков $\left[N_{1}^{j}, N_{2}^{j}\right]$ и $\left[L_{1}^{j}, L_{2}^{j}\right]$ соответственно. Из выпуклости $A$ вытекает, что $N_{3}^{j}, L_{3}^{j} \in A$, т.е. $\left[N_{3}^{j}, L_{3}^{j}\right] \subset A$. Очевидно, что отрезок $\left[N_{3}^{j}, L_{3}^{j}\right]$ имеет направление $l_{j}$ и длину $d(A)$. Покажем, что

$$
B^{*}=\operatorname{Cl}\left(\cup\left\{\left[N_{3}^{j}, L_{3}^{j}\right] \mid j=1,2, \ldots\right\}\right) \subset \mathscr{L}(A) .
$$

Из вьпуклости и замкнутости $A$ вытекает, что $B^{*} \subset A$. Остается показать, что $B^{*}-$ тело постоянной ширины.

Легко видеть, что для того чтобы $\operatorname{diam}\left(B^{*}, l_{i}\right)=d(A)$ необходимо и достаточно, чтобы $B^{*} \subset T\left(N_{3}^{i}, L_{3}^{i}\right)$. Это в свою очередь будет выполняться, если для произвольных $i \neq j\left[N_{3}^{j}, L_{3}^{j}\right] \subset T\left(N_{3}^{i}, L_{3}^{i}\right)$. Поскольку $\left[N_{1}^{j}, L_{1}^{j}\right] \subset T\left(N_{1}^{i}, L_{1}^{i}\right)$ и $\left[N_{2}^{j}, L_{2}^{j}\right] \subset T\left(N_{2}^{i}, L_{2}^{i}\right)$, то, сдвинув первую картину на вектор $\frac{1}{2}\left(N_{2}^{i}-N_{1}^{i}\right)=\frac{1}{2}\left(L_{2}^{i}-L_{1}^{i}\right)$, а вторую - на противоположньй вектор $\frac{1}{2}\left(N_{1}^{i}-N_{2}^{i}\right)$, получим

$$
\begin{aligned}
& {\left[N_{1}^{j}+\frac{1}{2}\left(N_{2}^{i}-N_{1}^{i}\right), L_{1}^{j}+\frac{1}{2}\left(N_{2}^{i}-N_{1}^{i}\right)\right] \subset T\left(N_{1}^{i}, L_{1}^{i}\right)+\frac{1}{2}\left(N_{2}^{i}-N_{1}^{i}\right)=T\left(N_{3}^{i}, L_{3}^{i}\right),} \\
& {\left[N_{2}^{j}-\frac{1}{2}\left(N_{2}^{i}-N_{1}^{i}\right), L_{2}^{j}-\frac{1}{2}\left(N_{2}^{i}-N_{1}^{i}\right)\right] \subset T\left(N_{2}^{i}, L_{2}^{i}\right)-\frac{1}{2}\left(N_{1}^{i}-N_{2}^{i}\right)=T\left(N_{3}^{i}, L_{3}^{i}\right) .}
\end{aligned}
$$

Поскольку множество $T\left(N_{3}^{i}, L_{3}^{i}\right)$ вьпукло, оно содержит и середины отрезков

$$
\left[N_{1}^{j}+\frac{1}{2}\left(N_{2}^{i}-N_{1}^{i}\right), N_{2}^{j}-\frac{1}{2}\left(N_{2}^{i}-N_{1}^{i}\right)\right] \quad \text { и } \quad\left[L_{1}^{j}+\frac{1}{2}\left(N_{2}^{i}-N_{1}^{i}\right), L_{2}^{j}-\frac{1}{2}\left(N_{2}^{i}-N_{1}^{i}\right)\right],
$$

точки $N^{\prime}$ и $L^{\prime}$ соответственно. Но

$$
\begin{aligned}
N^{\prime} & =N_{1}^{j}+\frac{1}{2}\left(N_{2}^{i}-N_{1}^{i}\right)+\frac{1}{2}\left[\left(N_{2}^{j}+\frac{1}{2}\left(N_{2}^{i}-N_{1}^{i}\right)\right)-\left(N_{1}^{j}-\frac{1}{2}\left(N_{2}^{i}-N_{1}^{i}\right)\right)\right] \\
& =N_{1}^{j}+\frac{1}{2}\left(N_{2}^{i}-N_{1}^{i}\right)+\frac{1}{2}\left(N_{2}^{j}-N_{1}^{j}\right)-\frac{1}{2}\left(N_{2}^{i}-N_{1}^{i}\right)=N_{1}^{j}+\frac{1}{2}\left(N_{2}^{j}-N_{1}^{j}\right)=N_{3}^{j}
\end{aligned}
$$

и, аналогично, $L^{\prime}=L_{3}^{j}$. Значит, $\left[N_{3}^{j}, L_{3}^{j}\right] \subset T\left(N_{3}^{i}, L_{3}^{i}\right)$, что и требовалось доказать. Отсюда получаем, что $\operatorname{diam}\left(B^{*}, l\right)=d(A)$ для всех направлений $l$. Это завершает доказательство леммы.

Обозначим через $V^{*}\left(l_{1}\right)$ центр масс множества $V\left(l_{1}\right)$, а через $\left[N_{1}, L_{1}\right]$ - диаметр, которому принадлежит точка $V^{*}\left(l_{1}\right)$. Пусть $\mathscr{L}\left(A, l_{1}\right)$ - множество всех тел $B \subset A$ постоянной ширины $d(A)$, имеющих своим диаметром направления $l_{1}$ отрезок $\left[N_{1}, L_{1}\right]$.

Рассмотрим направление $l_{2}$. Для каждого тела $B \in \mathscr{L}\left(A, l_{1}\right)$ зафиксируем точку $V\left(B, l_{2}\right)$ - центр диаметра направления $l_{2}$. Согласно лемме множество $V\left(l_{1}, l_{2}\right)=$ $\left\{V\left(B, l_{2}\right) \mid B \in \mathscr{L}\left(A, l_{1}\right)\right\}$ является вьпуклым замкнутым множеством с пустой внутренностью. Обозначим через $V^{*}\left(l_{1}, l_{2}\right)$ центр масс этого множества, а через $\left[N_{2}, L_{2}\right]$ - диаметр направления $l_{2}$, которому принадлежит точка $V^{*}\left(l_{1}, l_{2}\right)$. Через $\mathscr{L}\left(A ; l_{1}, l_{2}\right)$ обозначим множество всех тел $B \in \mathscr{L}\left(A, l_{1}\right)$, содержащих диаметр $\left[A_{2}, B_{2}\right]$.

Далее проводим построение по индукции. Предположим, что мы уже рассмотрели направления $l_{1}, \ldots, l_{n-1}$, где $n \geqslant 2$. Для каждого $B \in \mathscr{L}\left(A ; l_{1}, \ldots, l_{n-1}\right)$ зафиксируем точку $V\left(B, l_{n}\right)$ - центр диаметра направления $l_{n}$. Пусть $V^{*}\left(l_{1}, \ldots, l_{n}\right)$ - центр масс 
множества $V\left(l_{1}, \ldots, l_{n}\right)=\left\{V\left(B, l_{n}\right) \mid B \in \mathscr{L}\left(A ; l_{1}, \ldots, l_{n-1}\right)\right\}$, а $\left[N_{n}, L_{n}\right]$ - диаметр направления $l_{n}$, содержаший точку $V^{*}\left(l_{1}, \ldots, l_{n}\right)$. Через $\mathscr{L}\left(A ; l_{1}, \ldots, l_{n}\right)$ обозначим множество всех тел $B \in \mathscr{L}\left(A ; l_{1}, \ldots, l_{n-1}\right)$, содержащих диаметр $\left[A_{n}, B_{n}\right]$.

По построению

$$
\mathscr{L}\left(A, l_{1}\right) \supset \mathscr{L}\left(A ; l_{1}, l_{2}\right) \supset \mathscr{L}\left(A ; l_{1}, l_{2}, l_{3}\right) \supset \cdots .
$$

Множество

$$
\cap\left\{\mathscr{L}\left(A ; l_{1}, l_{2}, \ldots, l_{n}\right) \mid n \in \mathbb{N}\right\}
$$

непусто и замкнуто в метрике Хаусдорфа в $\exp \mathbb{R}^{n}$, и все элементы этого множества обязаны содержать все диаметры $\left[N_{n}, L_{n}\right]$. Но эти диаметры берутся из всюду плотного множества направлений и поэтому однозначно определяют тело, содержащее их. Это единственное выпуклое тело мы обозначим через $r(A)$.

Очевидно, что определенное таким образом отображение $r: \mathscr{M} \rightarrow \mathscr{N}$ непрерывно и является ретракцией. Таким образом, пространство $\mathscr{N}$, являясь ретрактом AR-пространства $\mathscr{M}$, само является AR-пространством. По теореме Торуньчика $\mathscr{N}$ является $Q$-многообразием.

\section{СПИСОК ЦИТИРОВАННОЙ ЛИТЕРАТУРЫ}

[1] Nadler S., Quinn J. E., Stavrokas N. M. Hyperspaces of compact convex sets // Bull. Polish Acad. Sci. Math. 1977. V. 25. №4. P. 381-385.

[2] Базилевич Л.Е. Про одну екзотичну псевдовнутрішність у гіперпросторі опуклих компактів // Мат. методи і фіз.-мех. поля. 1991. Т. 34. С. 15-18.

[3] Bazylevych L. E. On the hyperspace of strictly convex bodies // Матем. Студі. 1993. Т. 2. C. $83-86$.

[4] Torunczyk H. CE-images of the Hilbert cube and characterization of $Q$-manifolds // Fund. Math. 1980. V. 106. № 1. P. 31-40. 